\title{
\#outubrorosa e promoção da saúde: análise das postagens no Instagram sobre o câncer de mama
}

\author{
\#outubrorosa and health promotion: analysis of Instagram posts about \\ breast cancer
}

\section{\#outubrorosa y promoción de la salud: análisis de las publicaciones en Instagram sobre cáncer de mama}

Marcela Carvalho Rodrigues ${ }^{1, a}$

marcelamcr@hotmail.com | https://orcid.org/0000-0002-3156-9692

Lucas França Garcia ${ }^{1, b}$

lucasfgarcia@gmail.com | https://orcid.org/0000-0002-5815-6150

Marcelo Picinin Bernuci, ${ }^{i, c}$

marcelo.bernuci@unicesumar.edu.br | https://orcid.org/o0oo-0003-2201-5978

${ }^{1}$ Universidade Cesumar, Instituto Cesumar de Ciência, Tecnologia e Inovação. Maringá, PR, Brasil.

a Mestrado em Promoção da Saúde pela Universidade Cesumar.

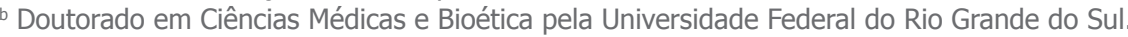

c Doutorado em Fisiologia pela Universidade de São Paulo.

\section{RESUMO}

O estudo apresentado neste artigo analisou a dinâmica das postagens sobre o tema da prevenção do câncer de mama no Instagram. As 300 postagens mais relevantes publicadas entre dezembro de 2019 e setembro de 2020 foram triadas a partir do uso da hashtag "\#outubrorosa". A maioria das postagens correspondia a fotos $(66,33 \%)$ com questões relacionadas à prevenção primária $(55,67 \%)$ que foram publicadas por não profissionais de saúde (45,33\%). As postagens apresentaram em média 196,06 curtidas e 14,43 comentários. Postagens com formato de foto publicadas por não profissionais de saúde foram as que apresentaram maior número de curtidas $(\mathrm{p}<\mathrm{0}, \mathrm{O1})$, já aquelas que abordaram o tema da prevenção primária foram as que apresentaram menor número de curtidas $(\mathrm{p}<\mathrm{0}, \mathrm{o1})$. Conclui-se que o Instagram constitui uma ferramenta importante a ser utilizada na conscientização da população jovem sobre autocuidado, porém se sugere a necessidade de avaliação da qualidade do conteúdo postado.

Palavras-chave: Redes sociais online; Promoção da Saúde; Saúde materno-infantil; Doenças crônicas não transmissíveis; Educação em saúde. 


\section{ABSTRACT}

The study presented in this article analyzed the dynamics of posts on the topic of the breast cancer prevention on Instagram. The 300 most relevant posts published between December 2019 and September 2020 were selected using the hashtag "\#outubrorosa". Most of the posts corresponded to photos (66.33\%) with questions related to primary prevention (55.67\%) and which were published by non-health professionals (45.33\%). The posts presented an average of 196.06 likes and 14.43 comments. Posts with photo format published by non-health professionals were the ones with the highest number of likes $(p<0.01)$, whereas those that addressed the topic of primary prevention were the ones with the least number of likes $(\mathrm{p}<0.01)$. It is concluded that Instagram is an important tool to be used in the awareness of the young population about self-care, however it is suggested the need to examine the quality of the posted content.

Keywords: Online social networks; Health promotion; maternal and child health; Chronic non-communicable diseases; Health education.

\section{RESUMEN}

El estudio presentado en este artículo analizó la dinámica de las publicaciones sobre el tema de la prevención del cáncer de mama en Instagram. Las 300 publicaciones más relevantes publicadas entre diciembre de 2019 y septiembre de 2020 fueron seleccionados proyectaron utilizando el hashtag "\#outubrorosa". La mayoría de las publicaciones correspondió a fotos $(66,33 \%)$ con preguntas relacionadas con la prevención primaria $(55,67 \%)$ y que fueron publicadas por personas que no son profesionales de la salud $(45,33 \%)$. Las publicaciones tuvieron un promedio de 196,06 likes y 14,43 comentarios. Las publicaciones con formato de foto publicadas por personas que no son profesionales de la salud fueron las que presentaron mayor número de gustas $(\mathrm{p}<\mathrm{0}, \mathrm{O} 1)$, mientras que las que abordaron el tema de prevención primaria fueron las que presentaron menor número de gustas $(\mathrm{p}<0,01)$. Se concluye que Instagram es una herramienta importante para ser utilizada en la concientización de la población joven sobre el autocuidado; sin embargo se sugiere la necesidad de evaluar la calidad del contenido publicado.

Palabras clave: Redes sociales en línea; Promoción de la salud; Salud maternal e infantil Enfermedades crónicas no transmisibles; Educación para la salud.

Contribuição dos autores:

Concepção e desenho do estudo: Marcela Carvalho Rodrigues, Lucas França Garcia e Marcelo Picinin Bernuci.

Aquisição, análise ou interpretação dos dados: Marcela Carvalho Rodrigues, Lucas França Garcia e Marcelo Picinin Bernuci.

Redação do manuscrito: Marcela Carvalho Rodrigues, Lucas França Garcia e Marcelo Picinin Bernuci.

Revisão crítica do conteúdo intelectual: Lucas França Garcia e Marcelo Picinin Bernuci.

Declaração de conflito de interesses: não há.

Fontes de financiamento: não houve.

Considerações éticas: não há.

Agradecimentos/Contribuições adicionais: não há.

Histórico do artigo: submetido: 5 maio 2021 | aceito: 27 jul. 2021 | publicado: 10 nov. 2021.

Apresentação anterior: não houve.

Licença CC BY-NC atribuição não comercial. Com essa licença é permitido acessar, baixar (download), copiar, imprimir, compartilhar, reutilizar e distribuir os artigos, desde que para uso não comercial e com a citação da fonte, conferindo os devidos créditos de autoria e menção à Reciis. Nesses casos, nenhuma permissão é necessária por parte dos autores ou dos editores. 


\section{INTRODUÇÃO}

O câncer de mama representa a principal doença maligna em mulheres em todo o mundo, cuja incidência tem aumentado consideravelmente nas últimas décadas (FITZMAURICE et al., 2019). Em 2020, cerca de 2,3 milhões de casos foram diagnosticados e 685.000 mortes foram notificadas (SUNG et al., 2021). Diferentemente do registrado em outras décadas, o câncer de mama tem acometido mulheres de todas as idades, com aumento constante dos casos entre as mais jovens ( $<40$ anos) (SOPIK, 2021). O câncer de mama em mulheres jovens apresenta um sério problema de saúde pública devido ao prognóstico relativamente ruim, em detrimento de maior prevalência de características tumorais desfavoráveis em comparação com o câncer de mama em idades mais avançadas, impactando com mais intensidade a expectativa de vida dessas jovens (KIM et al., 2018; CATHCART-RAKE et al., 2021).

Como esse tipo de câncer pode ser altamente evitável por meio de uma combinação de estratégias preventivas primárias ou secundárias (ROJAS; STUCKEY, 2016; YOULDEN et al., 2012), cujos fatores de risco modificáveis podem sofrer intervenção por meio de educação em saúde (BRITT; CUZICK; PHILLIPS, 2020), as iniciativas de saúde pública devem priorizar essas medidas a fim de amenizar os impactos impostos por essa enfermidade, notadamente entre jovens. Embora o fortalecimento das medidas de controle do câncer de mama tenha reduzido a taxa de mortalidade em muitos países, especialmente nos de alta renda, naqueles de média e baixa renda, como os da América Latina e Caribe e partes da Ásia, as taxas de mortalidade continuam a aumentar (AZAMJAH; SOLTAN-ZADEH; ZAYERI, 2019; RAMASWAMI et al., 2018; TORRE et al., 2016).

No Brasil, o câncer de mama ocupa a primeira posição entre os tipos de câncer mais frequentes, com estimativa de cerca de 66 mil novos casos para cada ano do triênio 2020-2022 (INCA, 2020). Esta malignidade tem apresentado uma taxa de mortalidade crescente na população feminina brasileira ao longo das décadas, em todos os grupos etários (20-44, 45-64 e 65+ anos), ocupando, no momento, a principal causa de morte de mulheres no país (LIMA et al., 2020). Ademais, tem sido também reportado um incremento significativo tanto na incidência quanto na taxa de mortalidade do câncer de mama em mulheres jovens na maioria dos estados brasileiros (ORLANDINI et al., 2021; SANTOS et al., 2013; SILVA et al., 2020). Para essas jovens, as altas taxas de mortalidade estão associadas a limitações de condição de vida, como renda reduzida e baixa escolaridade (SILVA, 2021), destacando a influência das inequidades sociais do país no perfil epidemiológico do câncer de mama.

A preocupação do governo brasileiro com o cenário epidemiológico imposto pelo câncer de mama existe desde a década de 1980, com a implementação de ações específicas como o programa Viva Mulher de 2005 e o Plano de Ações Estratégicas para o Enfrentamento das Doenças Crônicas Não Transmissíveis de 2011 (INCA, 2014). Estas ações reconhecem a importância da detecção precoce para o controle da malignidade, investindo ao longo das últimas décadas tanto no aumento do número de mamógrafos disponíveis nos serviços públicos, como na implementação de estratégias de conscientização da população para a importância da prevenção, como a campanha do Outubro Rosa (OHL et al., 2016).

Essa última, em especial, se ancora nas evidências científicas que apontam as estratégias de incentivo aos comportamentos de autocuidado em saúde, especialmente àquelas relacionadas à prevenção e diagnóstico precoce, como decisivas para um alcance global do controle do câncer de mama (ANASTASI; LUSHER, 2019; ASSIS; SANTOS; MIGOWSKI, 2020; GUTIÉRREZ; ALMEIDA, 2017; O'MAHONY et al., 2017; OMRANI et al., 2020). De fato, as ações de educação em saúde são consideradas como importantes ferramentas a serem articuladas nas estratégias de promoção da saúde da população (KICKBUSCH; NUTBEAM, 2017; SMITH; TANG; NUTBEAM, 2006; WHITEHEAD; TAKET; SMITH, 2003), especialmente quando conduz os indivíduos e as comunidades a desenvolverem maior controle sobre sua própria saúde, mobilizando 
recursos pessoais e sociais que extrapolam o setor da saúde (CHIAPPERINO; TENGLAND, 2015; TENGLAND, 2016).

Essa ideia foi sistematizada na década de 1980, na Carta de Ottawa que definiu a promoção da saúde e delineou os princípios-chave para a ação global em saúde, incluindo a importância de defender, capacitar e mediar a igualdade na saúde (ORGANIZAÇÃO MUNDIAL DA SAÚDE, 1986). No Brasil, com a implementação da Política Nacional de Promoção da Saúde (PNPS), as ações de promoção da saúde se articulam com a atenção primária à saúde, notadamente no que tange às estratégias de prevenção das doenças crônicas não transmissíveis, configurando-se na atualidade como protagonista no processo de construção de melhoria da qualidade de vida da população (BUSS et al., 2020; FORTUNE et al., 2018; BUSS; CARVALHO, 2007). Dessa forma, as estratégias de promoção da saúde baseadas em educação para saúde podem fortalecer o programa de controle do câncer de mama por meio do estímulo ao autocuidado em saúde, com foco especial nas ações de prevenção e de detecção precoce.

Estudos científicos têm apontado que o acesso das mulheres à informação qualificada sobre o câncer de mama promove o desenvolvimento de habilidades e competências de autocuidado, decisivo para melhoria do diagnóstico precoce desse tipo de câncer (LATEEF et al., 2020; NOMAN et al., 2020). A capacitação das mulheres sobre o processo saúde-doença melhora também o seu engajamento nos programas de prevenção, pois reduz o sentimento de incerteza sobre os fatores de risco e fortalece o acesso aos fatores de proteção (TELVIZIAN; MUKHERJI, 2020). Na última década, o acesso a informações foi facilitado pela incorporação das tecnologias digitais no cotidiano das pessoas, notadamente a partir de 2004, com o surgimento das redes sociais online (RSO), a chamada Web 2.0 ou Web Social (LUPTON, 2015; LUPTON; MASLEN, 2017). Sites de informação e suporte médico e de saúde, aplicativos e plataformas de RSO proliferaram, facilitando o acesso de leigos a informações relacionadas à saúde e proporcionando-lhes a oportunidade de compartilhar experiências de suas doenças ou atividades de promoção da saúde (SARTORI et al., 2020; YAMAGUCHI et al., 2020).

As plataformas de RSO, como Twitter, Facebook, YouTube e Instagram, além de permitirem o compartilhamento de informações, garantem aos usuários ambientes favoráveis para criação de seus próprios conteúdos, sendo, portanto, muito utilizadas para debater temas relacionados à saúde (FREE et al., 2013; GREKIN; BEATTY; ONDERSMA, 2019; YANG; VAN STEE, 2019), inclusive sobre o câncer de mama (DÖBRÖSSY et al., 2020). A utilização das RSO para incentivo da prática do autocuidado em saúde nas mulheres brasileiras não é utópica. Atualmente, mais de 160 milhões, dos quase 213,3 milhões de habitantes do país utilizam a internet, e mais de 70,3\% deles possuem contas em RSO (KEMP, 2021). Além disso, estudos prévios de nosso grupo de pesquisa têm demonstrado que o uso das RSO para debater assuntos relacionados tanto ao câncer de mama quanto ao câncer do colo do útero tem boa aceitabilidade entre as mulheres (BILOTTI et al., 2017, 2020; PEREIRA et al., 2020; SANTOS; SOUZA, 2019).

Dentre as RSO favoritas dos brasileiros, destaca-se o Instagram, com cerca de 928,5 milhões de usuários, tendo o Brasil o terceiro maior número de 'instagramers', atrás apenas dos Estados Unidos e da Índia (KEMP, 2021). Embora a popularidade do Instagram entre os brasileiros possa sugerir a viabilidade de sua articulação em estratégias de educação em saúde, poucos estudos têm sido direcionados para essa área. Até o momento, os resultados têm chamado atenção para a baixa qualidade do conteúdo sobre saúde postado pelos instagramers brasileiros, em diferentes contextos de saúde, como na epidemia do Zika vírus (CHANDRASEKARAN et al., 2017), na pandemia da covid-19 (GALHARDI et al., 2020), ou até mesmo sobre doenças específicas, como o câncer oral, por exemplo (PASSOS et al., 2020).

De todo modo, os resultados de estudos realizados em outros países têm demonstrado que os recursos do Instagram facilitam o estímulo de comportamentos de autocuidado em saúde (BOULOS; GIUSTINI; WHEELER, 2016; MARCON; BIEBER; AZAD, 2019; PILGRIM; BOHNET-JOSCHKO, 2019; PRICHARD 
et al., 2020), especialmente entre os jovens, como estímulo à atividade física (CURTIS et al., 2020) e a ingestão de alimentos mais saudáveis (GIL-QUINTANA; SANTOVEÑA-CASAL; ROMERO RIAÑO, 2021). No entanto, sua aplicabilidade no incentivo a comportamentos relacionados à prevenção e diagnóstico precoce do câncer de mama ainda não foi investigada. Até o momento, as evidências apenas apontam que o Instagram tem sido utilizado para debater questões mais relacionadas à experiência do convívio das mulheres com o câncer de mama (BASCH; MACLEAN, 2019).

Assim, diante do perfil epidemiológico do câncer de mama traçado ao longo dos últimos anos, marcado pelo aumento de diagnóstico em mulheres jovens, e da penatrabilidade das RSO no cotidiano dessa população, especialmente da plataforma Instagram, é possível hipotetizar que as postagens sobre o câncer de mama nessa RSO podem instigar comportamentos de autocuidado em saúde importantes para o fortalecimento das estratégias de controle desse tipo de câncer. Assim, o objetivo primordial do presente estudo foi analisar o conteúdo das publicações sobre o câncer de mama triados pela \#outubrorosa, a fim de compreender como esta campanha tem incentivado os instagramers a debater questões relacionadas ao controle dessa malignidade no Brasil. Acreditamos que os dados aqui apresentados possam subsidiar discussões futuras sobre a potencialidade do uso do Instagram em estratégias de educação em saúde baseadas no uso das tecnologias da informação e comunicação.

\section{METODOLOGIA}

Trata-se de um estudo descritivo de base quantitativa que analisou o conteúdo de postagens (posts) recuperadas por hashtags (\#) na RSO Instagram (Facebook, Menlo Park, CA). Obedeceu aos termos e condições de uso e política de privacidade do Instagram. Ele não foi registrado nem avaliado pelo sistema CEP/Conep conforme Inciso III, Parágrafo Único, do Artigo $1^{\circ}$ da Resolução 512 de 2016 do Conselho Nacional de Saúde, pois utilizou informações de acesso público, como prescrito nos termos da Lei $\mathrm{n}^{\circ}$ 12.527, de 18 de novembro de 2011. Todos os dados coletados não contêm informações de identificação para garantir o anonimato dos participantes e evitar o uso indevido das informações. O estudo seguiu procedimentos metodológicos semelhantes ao descrito em estudos prévios (KATZ; STALEY; ATTAI, 2020; MODAVE et al., 2019).

\section{Obtenção de dados}

Os dados foram coletados manualmente a partir de capturas de tela diretamente do Instagram (versão do site) por um pesquisador que utilizou uma conta anônima. As 300 postagens mais relevantes (segundo o Instagram) foram triadas na área de busca a partir do uso da hashtag \#outubrorosa. Essa hashtag foi selecionada por representar a campanha nacional de controle do câncer de mama no Brasil, conhecida como 'Outubro Rosa'. Os dados foram coletados entre dezembro de 2019 e setembro de 2020. Cada postagem foi codificada e armazenada em pastas para posterior análise.

\section{Análise de dados}

A análise das postagens foi realizada manualmente usando métodos de classificação indutiva e dedutiva (ELO; KYNGÄS, 2008). As postagens foram caracterizadas de acordo com: 1) recursos da mídia (imagem com ou sem texto, foto, vídeo); 2) tipo de conteúdo (prevenção primária, prevenção secundária, prevenção terciária, informativo e irrelevante); 3) tags de interação (curtidas, comentários e visualizações); e 4) descrição autodeclarada da identidade da conta que publicou a postagem (profissionais de saúde, não profissionais da saúde, empresas ou associações e celebridades). 
A análise do tipo de conteúdo das postagens foi determinada seguindo as recomendações de controle do câncer de mama vigentes no Brasil (MIGOWSKI et al., 2018). Considerou-se que os conteúdos relacionados à prevenção primária tratavam de ações realizadas para remover causas do câncer de mama e fatores de risco que aumentam a chance de que ele ocorra (por exemplo, obesidade, tabagismo etc.) bem como o estímulo de adesão a fatores protetivos (por exemplo, orientação de atividade física, alimentação saudável etc.). As medidas de prevenção secundária, por outro lado, tratavam de ações realizadas para a detecção precoce do câncer de mama (por exemplo, rastreamento de lesões precursoras por meio do exame clínico das mamas, confirmação diagnóstica pela mamografia). Já as medidas de prevenção terciária se direcionavam a discutir sobre o tratamento do câncer de mama, tanto local (cirurgia e radioterapia) quanto sistêmico (quimioterapia, hormonioterapia e terapia biológica).

Os conteúdos classificados como informativos indicavam ou notificavam datas específicas de campanhas de controle do câncer de mama. Aqueles classificados como 'irrelevantes' tratavam de outros aspectos não pertinentes a esse tipo de câncer, geralmente com associações espúrias.

Os dados foram analisados por dois pesquisadores independentes que classificaram todas as postagens. As análises foram comparadas entre os pesquisadores e aquelas que apresentaram discrepâncias foram analisadas por um terceiro pesquisador.

\section{Análise estatística}

Foi realizada uma análise descritiva dos resultados para a obtenção de gráficos e tabelas de frequência, com o intuito de realizar uma caracterização quantitativa dos dados. Deste modo, os resultados estão apresentados como frequência absoluta e relativa para as variáveis categórica, e média, mediana, desvio padrão, mínimo e máximo para as variáveis numéricas. A análise da relação das variáveis de interação dos usuários com as características das postagens foi realizada por meio de correlação bisserial de postos (rank biserial correlation) (CURETON, 1956). Todas as análises foram realizadas com o auxílio do ambiente estatístico R (R DEVELOPMENT CORE TEAM, 2015), versão 3.6.2.

\section{RESULTADOS}

\section{Caracterização das postagens}

A Tabela 1 apresenta os dados relativos à caracterização das postagens. Nota-se que entre as 300 postagens analisadas, o tipo de mídia mais frequente foi o de foto $(66,33 \%)$, seguido de imagens com texto (33,33\%). Em relação ao tema abordado nas postagens, destaca-se a prevenção primária, presente em mais da metade das postagens (55,67\%), e o de cunho informativo (39,33\%). Ainda, nota-se que $45,33 \%$ das postagens foram realizadas por contas autodeclaradas como não profissionais de saúde, enquanto 24,00\% por empresas ou associações. Exemplos dos tipos de postagens por tema de conteúdo abordado estão apresentados na Figura 1. 


\section{Tabela 1 - Distribuição de frequências das características das postagens}

\begin{tabular}{lcc} 
Variável & Frequência absoluta & $\%$ \\
Tipo de mídia* & & \\
\hline Imagem & 17 & $5,67 \%$ \\
Imagem com texto & 100 & $33,33 \%$ \\
Foto & 199 & $66,33 \%$ \\
Vídeo & 9 & $3,00 \%$ \\
Tema* & & \\
Prevenção primária & 167 & $55,67 \%$ \\
Prevenção secundária & 47 & $15,67 \%$ \\
Prevenção terciária & 22 & $7,33 \%$ \\
Informativo/ Educativo & 118 & $39,33 \%$ \\
\hline Identidade da conta & & \\
Profissionais de saúde & 41 & $13,67 \%$ \\
Não profissionais de saúde & 136 & $45,33 \%$ \\
Empresas ou associações & 72 & $24,00 \%$ \\
Celebridades & 7 & $2,33 \%$ \\
\hline
\end{tabular}

* Uma postagem pode ter mais de um formato, tema ou origem. Fonte: elaborada pelos autores.
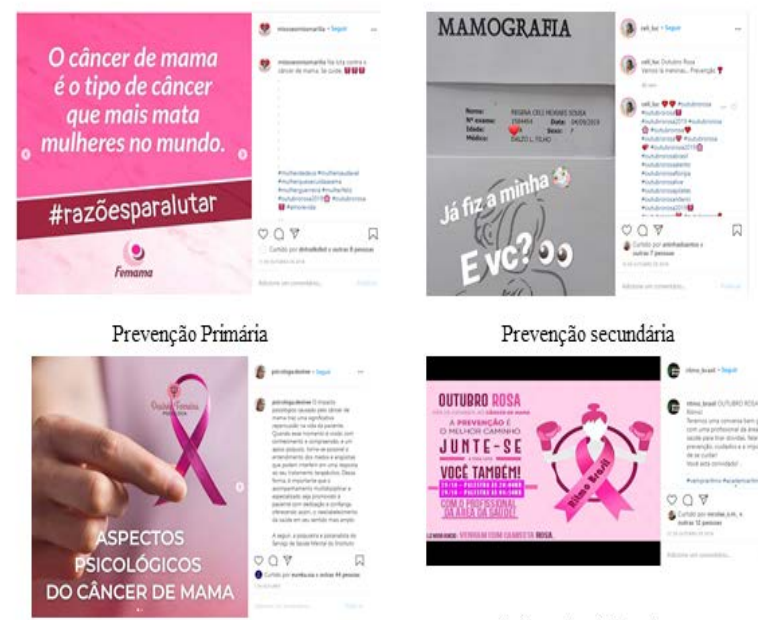

Prevenção Terciária

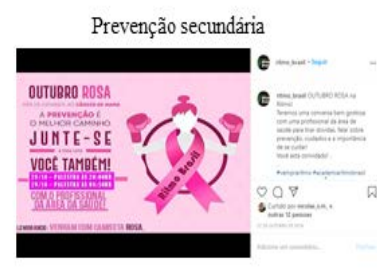

Informativo Educativo

Figura 1 - Representação dos tipos de postagens por tema abordado Fonte: prints feitos pelos autores.

\section{Caracterização da interação dos usuários}

Os dados referentes à caracterização da interação dos usuários com as postagens estão apresentados na Tabela 2. Nota-se que a informação a respeito do número de curtidas foi obtida para 292 das 300 postagens avaliadas, sendo que, em média, as postagens possuem 196,06 curtidas, embora essa quantidade apresente grande variabilidade, com desvio padrão de 642,70 curtidas, variando entre duas e 7.006 curtidas. Já em relação aos comentários, vê-se que as postagens possuem em média 14,43, com desvio padrão de 25,76, sendo que o número máximo observado entre as postagens foi de 215 comentários. Cabe ressaltar que tal informação foi obtida para 256 das 300 postagens. Por fim, nota-se que apenas nove postagens apresentaram 
o número de visualizações, com média de 497 e desvio padrão de 301,18 visualizações. Ainda, os números mínimo e máximo observados foram de 800 e 972 visualizações, respectivamente.

Tabela 2 - Caracterização das variáveis de interação do usuário

\begin{tabular}{lcccccc} 
Variável & n & Média & $\begin{array}{c}\text { Desvio } \\
\text { padrão }\end{array}$ & Mínimo & Mediana & Máximo \\
Curtidas & 292 & 196,06 & 642,70 & 2 & 70 & 7006 \\
Comentários & 256 & 14,43 & 25,76 & 1 & 6 & 215 \\
Visualizações & 9 & 497,00 & 301,18 & 80 & 567 & 972 \\
\hline
\end{tabular}

Fonte: elaborada pelos autores.

\section{Correlação das variáveis de interação com as características das postagens}

A Tabela 3 apresenta os dados relativos à correlação entre número de curtidas e características das postagens. Nota-se que postagens com formato de foto foram as que apresentaram a maior mediana de curtidas entre todos os formatos avaliados (84 curtidas), sendo a correlação entre as variáveis significativa (valor $\mathrm{p}<0,01$ ) e positiva (D de 0,13 ), o que indica que as que utilizaram esse formato tenderam a apresentar um maior número de curtidas. Apenas uma publicação no formato de vídeo apresentou o número de curtidas, sendo que nesse caso não foi possível avaliar a correlação. A correlação no caso do formato de imagem com texto também foi significativa (valor $\mathrm{p}<0,01$ ), mas negativa ( $\mathrm{D}$ de -0,11), sendo que a mediana do número de curtidas para esse formado foi de 43, pouco mais da metade do observado para as fotos, indicando que as postagens que utilizaram o formato de imagem com texto tenderam a apresentar um menor número de curtidas.

Em relação aos temas abordados nas postagens, nota-se que a menor mediana do número de curtidas foi observada para o tema referente à prevenção primária (53,50 curtidas), sendo ele o único tema que apresentou correlação significativa com a variável (valor $\mathrm{p}<0,01$ ), embora negativa (D de -0,12), ou seja, postagens que debateram o tema da prevenção primária tenderam a apresentar um menor número de curtidas.

Em relação à origem, cabe destacar que as duas correlações significativas foram com postagens originadas de pessoas comuns e de pessoas jurídicas (valores $\mathrm{p}<0,01$ ), sendo que em mediana, as postagens feitas por pessoas comuns apresentam um maior número de curtidas em relação às de pessoas jurídicas (medianas de 93,50 e 35,00 curtidas, respectivamente), indicando que as postagens publicadas por pessoas comuns tendem a receber maior número de curtidas.

Para as demais características, não há evidências amostrais de correlação significativa com o número de curtidas de acordo com os resultados do teste rank biserial correlation, no nível de $5 \%$ de significância. 
Tabela 3 - Correlação do número de curtidas com as características das postagens

\begin{tabular}{lcccc} 
Variável & \multicolumn{4}{c}{ Curtidas } \\
& Mediana & IIQ & D & Valor p \\
Tipo de mídia & & & \\
$\quad$ Imagem & 48,50 & $(18 ; 125)$ & $-0,02$ & 0,21 \\
Imagem com texto & 43,00 & $(18,75 ; 128)$ & $-0,11$ & $<0,01^{*}$ \\
Foto & 84,00 & $(46 ; 138)$ & 0,13 & $<0,01^{*}$ \\
Vídeo & 28,00 & - & - & - \\
Tema & & & & \\
$\quad$ Prevenção primária & 53,50 & $(25,75 ; 117)$ & $-0,12$ & $<0,01^{*}$ \\
Prevenção secundária & 82,00 & $(35 ; 180)$ & 0,02 & 0,40 \\
Prevenção terciária & 114,00 & $(39 ; 160)$ & 0,02 & 0,31 \\
Informativo/ Educativo & 66,00 & $(28 ; 158)$ & $-0,02$ & 0,53 \\
Identidade da conta & & & & \\
Profissionais de saúde & 56,00 & $(29,5 ; 156,5)$ & $-0,01$ & 0,72 \\
Não profissionais de saúde & 93,50 & $(46 ; 156)$ & 0,11 & $<0,01^{*}$ \\
Empresas ou associações & 35,00 & $(16 ; 66,5)$ & $-0,17$ & $<0,01^{*}$ \\
Celebridades & 64,00 & $(38 ; 85,5)$ & $-0,01$ & 0,45 \\
\hline
\end{tabular}

* Valor $p<0,05$

Fonte: elaborada pelos autores.

Os dados relativos à correlação entre número de comentários e características das postagens estão apresentados na Tabela 4. Nota-se que independentemente da característica das postagens, a mediana varia entre 3 e 7 comentários, sendo que nenhuma das características apresentou correlação significativa com o número de comentários, ao nível de $5 \%$ de significância.

Tabela 4-Correlação do número de comentários com as características das postagens

\begin{tabular}{lllll} 
Variável & $\begin{array}{l}\text { Comentários } \\
\text { Mediana }\end{array}$ & IIQ & D & Valor p \\
Tipo de mídia & & & & \\
$\quad$ Imagem & 7,00 & $(4 ; 13,5)$ & 0,01 & 0,66 \\
Imagem com texto & 7,00 & $(3 ; 14)$ & 0,02 & 0,60 \\
Foto & 6,00 & $(3 ; 14)$ & $-0,02$ & 0,56 \\
Vídeo & 5,00 & $(2,5 ; 12,5)$ & $-0,01$ & 0,69 \\
Tema & & & & \\
$\quad$ Prevenção primária & 7,00 & $(3 ; 12)$ & 0,02 & 0,57 \\
Prevenção secundária & 4,00 & $(1,5 ; 17,5)$ & $-0,02$ & 0,38 \\
Prevenção terciária & 5,50 & $(3,25 ; 11,75)$ & $-0,01$ & 0,74 \\
$\quad$ Informativo/ Educativo & 5,00 & $(2 ; 12)$ & $-0,03$ & 0,36 \\
Identidade da conta & & & & \\
$\quad$ Profissionais de saúde & 4,50 & $(2 ; 10,25)$ & $-0,04$ & 0,11 \\
Não profissionais de saúde & 6,00 & $(3 ; 15)$ & 0,04 & 0,32 \\
Empresas ou associações & 4,00 & $(2 ; 10)$ & $-0,05$ & 0,13 \\
Celebridades & 3,00 & $(2 ; 5,5)$ & $-0,01$ & 0,23 \\
\hline
\end{tabular}

* Valor $p<0,05$

Fonte: elaborada pelos autores. 


\section{DISCUSSÃO}

A plataforma de RSO Instagram é a predileta dos jovens brasileiros e tem sido muito investigada na área da saúde como ferramenta educativa diante do poder de influenciar comportamentos exercido pelos instagramers (FUNG et al., 2020). Como o estímulo do autocuidado em saúde depende de um bom conhecimento dos fatores ou processos envolvidos com a manutenção do estado de saúde ou da qualidade de vida, é de fundamental importância que as informações pertinentes a esses fatores sejam disseminadas e utilizadas para concretização de conhecimentos relevantes para a promoção da saúde (TENGLAND, 2010). Assim, se objetivou no presente estudo analisar a dinâmica das postagens no Instagram sobre o câncer de mama pelos brasileiros, a fim de inferir o potencial do uso dessa RSO em estratégias direcionadas para o controle dessa malignidade, especialmente entre a população jovem.

Referente à caracterização das postagens, identificou-se que o formato midiático utilizado com maior frequência pelos instagramers brasileiros para divulgar conteúdo sobre o câncer de mama foi o de foto. Além de ter sido a opção mais frequente, as postagens no formato de foto foram as que obtiveram o maior número de curtidas e visualizações. Como o Instagram é uma plataforma de RSO de compartilhamento de fotos e vídeos, é esperado que de fato o maior engajamento dos usuários seja com esse tipo de mídia, visto que as fotos permeiam a identidade do instagramer decisiva para manutenção de sua rede e chave no processo influenciador como já demonstrado em outros estudos (HABIBI; SALIM, 2021; SYCINSKADZIARNOWSKA et al., 2020). Entende-se que imagens como fotos, pictogramas e infográficos são importantes ferramentas para manutenção da identidade da conta e relação do seguidor com o conteúdo postado, visto que possuem potencial de impactar as atitudes e percepções acerca do que o instagramer pretende influenciar (BARROS et al., 2014; HOUTS et al., 2006). Além disso, no caso específico do câncer de mama, tem sido mostrado que informações da internet podem ser difíceis de serem lidas e interpretadas, e que a utilização de conteúdos estáticos, como fotos por exemplo, quando relacionado a essa temática, podem facilitar o engajamento do usuário (BASCH et al., 2019; HABIBI; SALIM, 2021). Resultados semelhantes ao aqui apresentado foram observados em outro estudo que analisou postagens do Instagram na língua inglesa referentes ao câncer de mama, no qual a maioria das postagens era também composta por fotos (BASCH; MACLEAN, 2019), o que sugere que a escolha desse tipo de mídia para divulgar o tema do câncer de mama no Instagram não é exclusiva dos brasileiros.

Entretanto, apesar do Instagram ser uma ferramenta que possibilita atingir muitas pessoas, as postagens de fotos estáticas podem não trazer informações suficientes para os usuários, fazendo com que eles sintam necessidade de migrar para outras plataformas em busca de conteúdos mais interativos, como criticado em outro estudo (BASCH et al., 2015). Assim, a plataforma Youtube, caracterizada pelo compartilhamento de vídeos, aparece como uma alternativa para aquisição de informações rápidas e dinâmicas sobre o câncer de mama (CLARKE HILLYER et al., 2019). Neste sentido, pressupõe-se que a utilização de vídeos com entrevistas e relatos pessoais pode gerar maior engajamento do público no Instagram, visto que isso foi identificado em outro estudo, cujo foco foi o diabetes (GABARRON et al., 2020). No entanto, no presente estudo, das 300 postagens sobre o tema do câncer de mama, apenas nove utilizaram o formato de vídeo, das quais apenas uma apresentou indicação de curtidas, dificultando assim a análise da importância desse tipo de mídia para o engajamento do usuário. De todo modo, com base no grande volume de interações (curtidas) obtidas nas postagens que usaram o formato de imagens estáticas (foto) demonstrado no presente estudo, é possível inferir que houve um bom engajamento dos seguidores com o conteúdo postado; entretanto, não se pode descartar a possibilidade do uso de mídias mais complexas, como os vídeos, capazes de movimentar com mais solidez o propósito da rede de sociabilização de conteúdos sobre saúde. 
Em relação ao conteúdo das postagens, observou-se que a maioria arremetia a algum aspecto da prevenção primária. A prevenção, de forma geral, representa ações cujo intuito primordial é reduzir as chances de um indivíduo contrair uma determinada doença (CZERESNIA; FREITAS, 2003) e, quando se arremete às questões ditas 'primárias', consiste em evitar fatores de risco predeterminados (INUMARU; SILVEIRA; NAVES, 2011). Nesta lógica, as postagens analisadas no presente estudo que se enquadram no tema da prevenção primária do câncer de mama, abordavam, em sua maioria, temas relacionados a ações com foco nos fatores de risco da doença. Esse fato assume grande importância para o tema da educação em saúde, pois demonstra uma tendência de direcionamento das ações para os fatores modificáveis, diferente do que tem sido apontado em outros estudos, cujo foco tem sido a divulgação de informações sobre aspectos da prevenção secundária (HOUGHTON; HOWLAND; MCDONALD, 2019). Reconhecidamente, para a prevenção primária das doenças, especialmente as crônicas não transmissíveis, as estratégias de educação em saúde direcionadas para fatores possivelmente modificáveis, como hábitos de vida, podem de fato ser uma estratégia mais efetiva para um controle populacional da doença (ETMINANI et al., 2020; ASKLAND et al., 2020; CRUZ-COBO; SANTI-CANO, 2020). O fortalecimento dos conhecimentos acerca dos fatores de risco e protetivos podem contribuir sobremaneira para a prevenção do câncer de mama, especialmente nas mulheres jovens (SIDDIG et al., 2021), cujas chances de prevenção são maiores, pois ainda não estão tão expostas a alguns fatores de risco, classicamente descritos para as mulheres mais velhas, como a exposição crônica a hormônios esteroides por exemplo.

Porém, o que chama a atenção é que esse tipo de conteúdo não foi o mais popular entre os seguidores; as postagens sobre a prevenção primária tenderam a receber um menor número de curtidas dos usuários, quando comparadas àquelas sobre prevenção secundária, por exemplo. Esse dado é relevante, uma vez que sugere uma maior valorização de temas mais discutidos no cotidiano das mulheres, como a mamografia, que dá mais importância à prevenção secundária. A análise de postagens sobre a prevenção de doenças, com foco especial em questões de prevenção secundária foi também levantada em outros estudos tanto para doenças não transmissíveis (PAIGE et al., 2017; CHO et al., 2018; LATHA et al., 2020; MAJMUNDAR et al., 2020) como transmissíveis (CADAXA; SOUSA; MENDONÇA, 2015; DEHLIN et al., 2019; KEARNEY et al., 2019; SANZ-LORENTE et al., 2018). Todos esses estudos apontaram que as RSO são ferramentas eficazes para promover a conscientização dos indivíduos sobre a importância da prevenção, desde que o foco das ações se encontre em fatores passíveis de serem modificados, como o estímulo à adesão aos fatores de proteção e a busca de evitar os de risco, como já apontado em um elegante estudo de revisão sistemática (HOUGHTON; HOWLAND; MCDONALD, 2019). Desse modo, o presente estudo mostra um dado positivo sobre a dinâmica de publicações de conteúdo sobre o câncer de mama por instagramers brasileiros, que têm a tendência de publicar conteúdos pertinentes à prevenção primária; mesmo que não seja o preferido dos usuários, é tema central das ações de educação em saúde destinadas ao controle populacional dessa malignidade.

Foi também observado que grande parte das postagens no Instagram sobre o tema do câncer de mama foram realizadas por pessoas comuns, e não por profissionais da área de saúde. Esse fato deve ser visto com parcimônia, pois em primeira instância se mostra muito positivo, uma vez que demonstra a mobilização em rede das pessoas comuns, fora do nicho da saúde, que se prontificam a difundir conteúdo informativo sobre uma determinada doença. No entanto, corre-se o risco de se propagar conteúdo equivocado ou incorreto, que pode trazer transtornos à vida do usuário da RSO e prejudicar o desenvolvimento e propagação de hábitos adequados de cuidado com a saúde. Nessa perspectiva, apesar das RSO constituírem uma ferramenta alternativa e promissora de educação em saúde, que possibilitam o alcance por muitas pessoas, é importante analisar a confiabilidade dos conteúdos compartilhados, visto que se forem de baixa qualidade, os efeitos globais podem ser bastante negativos (WANG et al., 2019). De fato, um estudo recente 
de análise de conteúdo publicado na RSO Pinterest, demonstrou alta incidência de informações incorretas relacionadas à prevenção e ao tratamento do câncer de mama (WILNER; HOLTON, 2020). Mesmo que haja poucos estudos, realizados até o momento, sobre o uso das RSO para a educação em saúde direcionada ao controle do câncer de mama, os estudos que analisaram disseminação de conteúdo na web sobre saúde têm chamado muito atenção dos pesquisadores e órgãos públicos para a disseminação de informações por pessoas não qualificadas (CHEN; WANG; PENG, 2018; DONZELLI et al., 2018; PEYSER et al., 2021). De todo modo, não se pode ignorar o achado de que foram as postagens publicadas por contas declaradas de não profissionais de saúde que obtiveram maior número de curtidas. Ademais, a análise de correlação demonstrou uma tendência de ter mais curtidas em postagens realizadas por não profissionais de saúde, do que empresas ou associações. Esse fato sugere que, para o Instagram, a figura do instagramer como influenciador de comportamento não depende de sua qualificação, mas sim de outros atributos.

Outro desafio refere-se à autenticidade dos dados publicados pois, considerando que as tecnologias de mídia social foram desenvolvidas recentemente, os processos de avaliação da veracidade do conteúdo ainda se encontram nos estágios iniciais de desenvolvimento (LEVAC; SULLIVAN, 2010). Tendo em conta que a característica do Instagram é a visualização rápida das imagens, torna-se difícil considerar que os usuários possam analisar criticamente o conteúdo (PAIGE et al., 2017). Uma das formas de se disseminar desinformações nas RSO é a utilização de evidências não comprovadas e anedóticas (relatos pessoais), muito comuns entre os instagramers (MASSEY et al., 2020). Como sugestão de tentativa de refinamento da qualidade das postagens, pode-se buscar identificar links de acesso às referências utilizadas pelos instagramers na confecção da postagem (KEARNEY et al., 2019). Atualmente, plataformas como o Facebook e o Instagram não desenvolveram regulamentos referentes à desinformação em saúde, exceto para a covid-19 (JENKINS et al., 2020), sugerindo a necessidade imediata de discussões sobre o tema.

Outro dado importante levantado no presente estudo diz respeito às associações de algumas características das postagens com a interação do usuário. Foi mostrado que postagens no formato de foto e que abordaram questões pertinentes à prevenção secundária foram as que geraram maior interação do usuário. O sucesso de uma campanha ou ação nas RSO é medido por meios que revelam seu alcance, suas impressões e seu engajamento. Esse último consiste no número total de interações dos usuários em uma postagem, seja por meio de cliques, emojis ou curtidas (PARK; REBER; CHON, 2016; ZARZYCKI, 2018). Um 'curtir' indica que a postagem foi percebida pelo usuário, entretanto sinaliza pouco comprometimento quando comparado a comentários e compartilhamentos, pois esses requerem ações adicionais e maior esforço cognitivo (KIM; YANG, 2017; YUKI, 2015). Além disso, as curtidas e os compartilhamentos são interações rápidas que podem evidenciar a concordância do usuário com o conteúdo postado (WOHN; CARR; HAYES, 2016). Essas interações são uma forma de comunicação, pela qual o usuário, com um simples click, oferece um feedback aos outros usuários (KAUR et al., 2019; WOHN; CARR; HAYES, 2016). Considerando que o maior número de postagens sobre o câncer de mama foi de fotos estáticas, já era esperado que esse tipo de mídia recebesse maior número de curtidas, visto que fotos possibilitam o compartilhamento de informação de forma rápida e intuitiva, gerando maior interação do que outros tipos de mídias (YANG et al., 2020). Resultados semelhantes também foram encontrados em uma intervenção de conscientização sobre a vacinação contra o HPV no Facebook (BRANDT et al., 2020), como também na análise da \#Antivaccination no Instagram (KIM; SONG; LEE, 2020) e ainda na análise de conteúdo relacionada ao cigarro eletrônico, também no Instagram (GAO et al., 2020), todos demonstrando o potencial da mídia do tipo foto como mobilizadora da rede.

Porém, quando se pensa em construção do saber, o debate de ideias, exposição de dúvidas e depoimentos de experiências, exposto no formato de comentários, parece ser mais relevante. Como foi aqui observado, as postagens de comentários não constituíram um recurso muito utilizado pelos usuários, demonstrando pouco 
aproveitamento desse espaço virtual para o aprofundamento das discussões. Por outro lado, reconhecendose o potencial influenciador exercido pelos instagramers em seus seguidores, as publicações de conteúdo informativo sobre a prevenção primária do câncer de mama sugerem uma importância dessa RSO no controle dessa malignidade. Mesmo que tenha ocorrido uma maior tendência de curtidas de conteúdos de prevenção secundária, é importante reconhecer que o tema do câncer de mama tem repercutido pela rede, e que como a hashtag de busca utilizada no presente estudo foi a \#outubrorosa, a tendência dos usuários a valorizarem informações mais pertinentes à prevenção secundária é condizente com o propósito da campanha do Outubro do Rosa do Ministério da Saúde do Brasil, cujos conteúdos sobre a detecção precoce são mais frequentes. Entender sobre os tipos de conteúdo que são atraentes para determinados públicos é importante para aumentar esse engajamento (BONSÓN; ROYO; RATKAI, 2015), pois alguns conteúdos podem ser mais atraentes que os outros (BONSÓN; BEDNÁROVÁ, 2018), e saber qual é o mais atrativo pode facilitar o delineamento da estratégia educativa. Além disso, para que se consiga maior engajamento da população, é preciso ir além do compartilhamento de informações (MERGEL, 2013), a comunicação unilateral não aumenta a interação do usuário, (ZAVATTARO; SEMENTELLI, 2014), é preciso identificar a preferência do usuário antes de se executar a estratégia de mobilização via RSO (CHEN et al., 2020).

Assim, neste estudo observou-se correlação entre a interação e as características das postagens, e identificou-se que, ao comparar os temas da postagem, a prevenção secundária foi a temática que recebeu o maior número de curtidas, sendo o único tema que apresentou correlação significativa com essa variável. Esse resultado também foi encontrado em postagens sobre diabetes, nas quais conteúdos sobre prevenção secundária, enfatizados em dias de conscientização e outras comemorações, apresentaram maior número de curtidas (GABARRON et al., 2020). Outro estudo apontou que postagens na RSO chinesa Sina Weibo sobre prevenção da covid-19 geraram maior número de comentários e curtidas (NGAI et al., 2020). Além disso, a análise de fotos do Instagram com a hashtag \#zika apontou que a maioria das curtidas estava relacionada à temática de prevenção e transmissão da doença (SELTZER et al., 2017). Essa maior interação do público com postagens com a temática da prevenção pode ser explicada pela preferência dos usuários por conteúdos positivos (KITE et al., 2018), que despertem emoções positivas (CHO et al., 2018; NGAI; SINGH; LU, 2020) não relacionadas a evolução ou tratamento da doença. Um estudo recente de revisão demonstrou que intervenções que buscam melhorar o engajamento das pessoas no rastreamento e o diagnóstico precoce do câncer precisam, de fato, focar em conteúdos ligados aos fatores modificáveis (PLACKETT et al., 2020). Em conjunto, essas evidências sugerem que o foco das postagens de relevância para o controle do câncer de mama deve, de fato, se basear na prevenção primária, como o apresentado no presente estudo.

\section{CONSIDERAÇÕES FINAIS}

Este estudo teve como objetivo analisar a dinâmica das 300 postagens mais relevantes publicadas no Instagram sobre o câncer de mama em língua portuguesa do Brasil, com foco especial na campanha nacional de controle desta malignidade, conhecida como 'Outubro Rosa'. Com base nos dados aqui apresentados é possível concluir que os instagramers brasileiros têm tratado o tema do câncer de mama por meio de diferentes mídias e diferentes abordagens. A dinâmica das publicações tem privilegiado mídias estáticas, como fotos e imagens com texto, e conteúdos relacionados especialmente à prevenção primária do câncer de mama. No entanto, no que diz respeito à interação com os usuários, os conteúdos de cunho informativo sobre a prevenção secundária, publicados no formato de fotos e em contas autodeclaradas como de não profissionais da área de saúde, foram os que tenderam a ter maior popularidade. Esse dado levanta questionamentos importantes sobre a confiabilidade da fonte de informação, visto que os comportamentos de autocuidado em saúde podem ser influenciados pelo conteúdo das postagens. A publicação de notícias falsas nas RSO sobre temas da área de saúde tem sido cada vez mais frequente, 
e caracterizada como uma 'infodemia' que prejudica a eficiência das estratégias de controle de muitas enfermidades, especialmente aquelas potencialmente evitáveis, como o câncer de mama. Como aqui apresentado, as postagens publicadas por indivíduos não especializados no tema apresentaram amplo alcance e engajamento, demonstrando fragilidade nas estratégias de comunicação adotadas pelos profissionais da área de saúde, além de evidenciar limitações de letramento digital do usuário. De todo modo, se reconhece a iniciativa positiva dos instagramers brasileiros de debaterem o tema do câncer de mama, especialmente no que tange a questões pertinentes à prevenção, primordial para o melhor controle dessa malignidade; porém, os riscos da disseminação de conteúdo informativo de qualidade limitada colocam em xeque o potencial dessa estratégia.

\section{REFERÊNCIAS}

ANASTASI, Natasha; LUSHER, Joanne. The impact of breast cancer awareness interventions on breast screening uptake among women in the United Kingdom: a systematic review. Journal of Health Psychology, v. 24, n. 1, p. 113-124, 2019. DOI: https://doi.org/10.1177\%2F1359105317697812. Disponível em: https:// journals.sagepub.com/doi/10.1177/1359105317697812. Acesso em: 17 set. 2021.

ASKLAND, Kathleen et al. Educational, supportive and behavioural interventions to improve usage of continuous positive airway pressure machines in adults with obstructive sleep apnoea. The Cochrane Database of Systematic Reviews, Oxford, v. 4, n. 4, p. CD007736, 2020, DOI: https:// doi.org/10.1002/14651858.cd007736.pub3. Disponível em: https://www.ncbi.nlm.nih.gov/pmc/articles/ PMC7137251/pdf/CD007736.pdf. Acesso em: 21 set. 2021.

ASSIS, Mônica de; SANTOS, Renata Oliveira Maciel dos; MIGOWSKI, Arn. Detecção precoce do câncer de mama na mídia brasileira no Outubro Rosa. Physis: Revista de Saúde Coletiva, Rio de Janeiro, v. 30, n. 1 , p. 1-20, 2020. DOI: https://doi.org/10.1590/S0103-73312020300119. Disponível em: https://www.scielo.br/j/ physis/a/yv3nLJmpv55Jtk8nshYXHBM/?lang=pt. Acesso em: 17 set. 2021.

AZAMJAH, Nasrindokht; SOLTAN-ZADEH, Yasaman; ZAYERI, Farid. Global trend of breast cancer mortality rate: a 25-year study. Asian Pacific Journal of Cancer Prevention, Bangkok, v. 20, n. 7, p. 2015-2020, 2019. DOI: https://dx.doi.org/10.31557\%2FAPJCP.2019.20.7.2015. Disponível em: http://journal.waocp.org/ article_88661.html. Acesso em: 17 set. 2021.

BARROS, Izadora M. C. et al. The use of pictograms in the health care: a literature review. Research in Social and Administrative Pharmacy, New York, v. 10, n. 5, p. 704-719, 2014. DOI: https://doi. org/10.1016/j.sapharm.2013.11.002. Disponível em: https://www.sciencedirect.com/science/article/abs/pii/ S155174111300243X?via\%3Dihub. Acesso em: 20 set. 2021.

BASCH, Corey H. et al. Characteristics of YouTube ${ }^{\mathrm{TM}}$ videos related to mammography. Journal of Cancer Education, New York, v. 30, n. 4, p. 699-703, 2015. DOI: https://doi.org/10.1007/s13187-014-0769-9. Disponível em: https://link.springer.com/article/10.1007\%2Fs13187-014-0769-9. Aceso em: 20 set. 2021.

BASCH, Corey H. et al. Readability of online breast cancer information. The Breast Journal, [s. I.], v. 25, n. 3, p. 562-563, 2019. DOI: https://doi.org/10.1111/tbj.13276. Disponível em: https://onlinelibrary.wiley.com/ doi/10.1111/tbj.13276. Acesso em: 20 set. 2021.

BASCH, Corey H.; MACLEAN, Sarah A. Breast cancer on Instagram: a descriptive study. International Journal of Preventive Medicine, Isfahan, v. 10, p. 166, 2019. DOI: https://dx.doi.org/10.4103\%2Fijpvm. IJPVM 36 19. Disponível em: https://www.ijpvmjournal.net/article.asp?issn=2008-7802; year=2019; volume=10; issue $=1 ;$ spage $=166 ;$ epage $=166$; aulast=Basch. Acesso em: 20 set. 2021.

BILOTTI, Carolina Correia et al. m-Health no controle do câncer de colo do útero: pré-requisitos para o desenvolvimento de um aplicativo para smartphones. Revista Eletrônica de Comunicação, Informação e Inovação em Saúde, Rio de Janeiro, v. 11, n. 2, p. 1-18, 2017. DOI: https://doi.org/10.29397/reciis.v11i2.1217. Disponível em: https://www.reciis.icict.fiocruz.br/index.php/reciis/article/view/1217. Acesso em: 17 set. 2021.

BILOTTI, Carolina Correia et al. Aparelhos celulares e a detecção precoce do câncer de mama: possibilidade de educação em saúde para mulheres. Revista Família, Ciclos de Vida e Saúde no Contexto Social, Uberaba, v. 8, n. 3, p. 370-382, 2020. DOI: https://doi.org/10.18554/refacs.v8i3.3933. Disponível em: http:// seer.uftm.edu.br/revistaeletronica/index.php/refacs/article/view/3933. Acesso em: 20 set. 2021. 
BONSÓN, Enrique; BEDNÁROVÁ, Michaela. The use of YouTube in western European municipalities. Government Information Quarterly, [s. I.], v. 35, n. 2, p. 223-232, 2018. DOI: http://dx.doi. org/10.1016/j.giq.2018.04.001. Disponível em: https://www.sciencedirect.com/science/article/abs/pii/ S0740624X18301989?via\%3Dihub. Acesso em: 20 set. 2021.

BONSÓN, Enrique; ROYO, Sonia; RATKAI, Melinda. Citizens' engagement on local governments' Facebook sites. An empirical analysis: The impact of different media and content types in Western Europe. Government Information Quarterly, [s. I.], v. 32, n. 1, p. 52-62, 2015. DOI: https://doi.org/10.1016/i.giq.2014.11.001. Disponível em: https://www.sciencedirect.com/science/article/abs/pii/S0740624X14001567. Acesso em: 20 set. 2021.

BOULOS, Maged N. Kamel; GIUSTINI, Dean M.; WHEELER, Steve. Instagram and WhatsApp in health and healthcare: an overview. Future Internet, [s. I.], v. 8, n. 3, p. 37, 2016. DOI: http://dx.doi.org/10.3390/ fi8030037. Disponível em: https://www.mdpi.com/1999-5903/8/3/37. Acesso em: 17 set. 2021.

BRANDT, Heather M. et al. Evaluating a technology-mediated HPV vaccination awareness intervention: a controlled, quasi-experimental, mixed methods study. Vaccines, Basel, v. 8, n. 4, p. 749, 2020. DOI: https://doi. org/10.3390/vaccines8040749. Disponível em: https://www.mdpi.com/2076-393X/8/4/749. Acesso em: 20 set. 2021.

BRITT, Kara L.; CUZICK, Jack; PHILLIPS, Kelly-Anne. Key steps for effective breast cancer prevention. Nature Reviews Cancer, Londres, v. 20, n. 8, p. 417-436, 2020. DOI: https://doi.org/10.1038/s41568-020-0266-x. Disponível em: https://www.nature.com/articles/s41568-020-0266-x. Acesso em: 17 set. 2021.

BUSS, Paulo Marchiori et al. Promoção da saúde e qualidade de vida: uma perspectiva histórica ao longo dos últimos 40 anos (1980-2020). Ciência \& Saúde Coletiva, Rio de Janeiro, v. 25, n. 12, p. 4723-4735, 2020. DOI: https://doi.org/10.1590/1413-812320202512.15902020. Disponível em: https://www.scielo.br/j/ csc/a/5BJghnvvZyB7GmyF7MLjgDr/abstract/?lang=pt. Acesso em: 17 set. 2021.

BUSS, Paulo Marchiori; CARVALHO, Antônio Ivo de. Health promotion in Brazil. Promotion \& Education, Londres, v. 14, n. 4, p. 209-213, 2007. DOI: https://doi.org/10.1177\%2F10253823070140040501. Disponível em: https://journals.sagepub.com/doi/abs/10.1177/10253823070140040501. Acesso em: 17 set. 2021.

CADAXA, Aedê Gomes; SOUSA, Maria de Fátima; MENDONÇA, Ana Valéria Machado. Conteúdos promotores de saúde em campanhas de Aids no Facebook dos ministérios da saúde do Brasil e do Peru. Revista Panamericana de Salud Publica, Washington, D. C., v. 38, n. 6, p. 457-463, 2015. Disponível em: https://www.scielosp.org/article/rpsp/2015.v38n6/457-463/. Acesso em: 20 set. 2021.

CATHCART-RAKE, Elizabeth J. et al. Breast cancer in adolescent and young adult women under the age of 40 years. JCO Oncology Practice, [s. I.], v. 17, n. 6, p. 305-315, 2021. DOI: https://doi.org/10.1200/OP.20.00793. Disponível em: https://ascopubs.org/doi/10.1200/OP.20.00793. Acesso em: 17 set. 2021.

CHANDRASEKARAN, Neeraja et al. The utility of social media in providing information on zika virus. Cureus, Palo, Alto, v. 9, n. 10, p. e1792, 2017. DOI: https://dx.doi.org/10.7759\%2Fcureus.1792. Disponível em: https:// www.cureus.com/articles/8571-the-utility-of-social-media-in-providing-information-on-zika-virus. Acesso em: 17 set. 2021.

CHEN, Liang; WANG, Xiaohui; PENG, Tai-Quan. Nature and diffusion of gynecologic cancer-related misinformation on social media: analysis of tweets. Journal of Medical Internet Research, Toronto, v. 20, n. 10, p. e11515, 2018. DOI: https://doi.org/10.2196/11515. Disponível em: https://www.jmir.org/2018/10/e11515/. Acesso em: 20 set. 2021.

CHEN, Qiang et al. Unpacking the black box: how to promote citizen engagement through government social media during the COVID-19 crisis. Computers in Human Behavior, New York, v. 110, p. 106380, 2020. DOI: https://doi.org/10.1016/j.chb.2020.106380. Disponivel em: https://www.sciencedirect.com/science/article/abs/ pii/S0747563220301333?via\%3Dihub. Acesso em: 20 set. 2021.

CHIAPPERINO, Luca; TENGLAND, Per-Anders. Empowerment in healthcare policy making: three domains of substantive controversy. Health Promotion Journal of Australia, West Perth, v. 26, n. 3, p. 210-215, 2015. DOI: https://doi.org/10.1071/HE15035. Disponível em: https://onlinelibrary.wiley.com/doi/abs/10.1071/HE15035. Acesso em: 17 set. 2021.

$\mathrm{CHO}$, Hyunyi et al. Visual cancer communication on social media: an examination of content and effects of \#melanomasucks. Journal of Medical Internet Research, Toronto, v. 20, n. 9, p. e10501, 2018. DOI: https:// dx.doi.org/10.2196\%2F10501. Disponível em : https://www.jmir.org/2018/9/e10501/. Acesso em: 20 set. 2021. 
CLARKE HILLYER, Grace et al. YouTube videos as a source of information about mastectomy. The Breast Journal, [s. I.], v. 25, n. 2, p. 349-350, 2019. DOI: https://doi.org/10.1111/tbj.13222. Disponível em: https:// onlinelibrary.wiley.com/doi/full/10.1111/tbj.13222. Acesso em: 20 set. 2021.

CRUZ-COBO, Celia; SANTI-CANO, M. José. Efficacy of diabetes education in adults with diabetes mellitus type 2 in primary care: a systematic review. Journal of Nursing Scholarship, Indianapolis, v. 52, n. 2, p. 155163, 2020. DOI: https://doi.org/10.1111/jnu.12539. Disponível em: https://sigmapubs.onlinelibrary.wiley.com/doi/ epdf/10.1111/inu.12539. Acesso em: 21 set. 2021.

CURETON, Edward E. Rank biserial correlation. Psychometrika, [s. I.], v. 21, p. 287-290, 1956. DOI: https:// doi.org/10.1007/BF02289138. Disponível em: https://link.springer.com/article/10.1007/BF02289138. Acesso em: 20 set. 2021.

CURTIS, Rachel G. et al. Can Instagram be used to deliver an evidence-based exercise program for young women? A process evaluation. BMC Public Health, [s. I.], v. 20, p. 1506, 2020. DOI: https://doi.org/10.1186/ s12889-020-09563-y. Disponível em: https://bmcpublichealth.biomedcentral.com/articles/10.1186/s12889-02009563-y. Acesso em: 20 set. 2021.

CZERESNIA, Dina; FREITAS, Carlos Machado de (org.). Promoção da saúde: conceitos, reflexões, tendências. Rio de Janeiro: Editora Fiocruz, 2003.

DEHLIN, Jessica M. et al. \#PrEP4Love: an evaluation of a sex-positive HIV prevention Campaign. JMIR Public Health and Surveillance, Toronto, v. 5, n. 2, p. e12822, 2019. DOI: https://doi.org/10.2196/12822. Disponível em: https://publichealth.jmir.org/2019/2/e12822/. Acesso em: 20 set. 2021.

DÖBRÖSSY, Bence et al. "Clicks, likes, shares and comments" a systematic review of breast cancer screening discourse in social media. PloS One, São Francisco, v. 15, n. 4, p. e0231422, 2020. DOI: https://doi. org/10.1371/journal.pone.0231422. Disponível em: https://journals.plos.org/plosone/article?id=10.1371/journal. pone.0231422. Acesso em: 17 set. 2021

DONZELLI, Gabriele et al. Misinformation on vaccination: a quantitative analysis of YouTube videos. Human Vaccines \& Immunotherapeutics, Austin, v. 14, n. 7, p. 1654-1659, 2018. DOI: https://doi.org/10.1080/216 45515.2018.1454572. Disponível em: https://www.tandfonline.com/doi/full/10.1080/21645515.2018.1454572. Acesso em: 21 set. 2021

ELO, Satu; KYNGÄS, Helvi. The qualitative content analysis process. Journal of Advanced Nursing, Oxford, v. 62, n. 1, p. 107-115, 2008. DOI: https://doi.org/10.1111/j.1365-2648.2007.04569.x. Disponível em: https:// onlinelibrary.wiley.com/doi/10.1111/j.1365-2648.2007.04569.x. Acesso em: 20 set. 2021.

ETMINANI, Kobra et al. How behavior change strategies are used to design digital interventions to improve medication adherence and blood pressure among patients with hypertension: systematic review. Journal of Medical Internet Research, Toronto, v. 22, n. 4, p. e17201, 2020, DOI: https://doi.org/10.2196/17201. Disponível em: https://www.jmir.org/2020/4/e17201/PDF. Acesso em: 21 set. 2021.

FITZMAURICE, Christina et al. Global, regional, and national cancer incidence, mortality, years of life lost, years lived with disability, and disability-adjusted life-years for 29 cancer groups, 1990 to 2017. JAMA Oncology, Chicago, v. 5, n. 12, p. 1749-1768, 2019. DOI: https://doi.org/10.1001/jamaoncol.2019.2996. Disponível em: https://jamanetwork.com/journals/jamaoncology/fullarticle/2752381. Acesso em: 16 set. 2021.

FORTUNE, K. et al. Health promotion and the agenda for sustainable development, WHO Region of the Americas. Bulletin of the World Health Organization, Genebra, v. 96, n. 9, p. 621-626, 2018. DOI: https:// dx.doi.org/10.2471\%2FBLT.17.204404. Disponível em: https://www.who.int/bulletin/volumes/96/9/17-204404. pdf. Acesso em: 17 set. 2021.

FREE, Caroline et al. The effectiveness of mobile-health technology-based health behaviour change or disease management interventions for health care consumers: a systematic review. PLoS Medicine, São Francisco, v. 10, n. 1, p. e1001362, 2013. DOI: https://doi.org/10.1371/journal.pmed.1001362. Disponível em: https:// journals.plos.org/plosmedicine/article?id=10.1371/journal.pmed.1001362. Acesso em: 17 set. 2021.

FUNG Isaac Chun-Hai et al. Public health implications of image-based social media: a systematic review of Instagram, Pinterest, Tumblr, and Flickr. The Permanente Journal, Portland, OR, v. 24, p. e18.307, 2020. DOI: https://doi.org/10.7812/tpp/18.307. Disponível em: https://www.ncbi.nlm.nih.gov/pmc/articles/PMC6907901/ pdf/18.307.pdf. Acesso em: 21 set. 2021. 
GABARRON, Elia et al. Factors engaging users of diabetes social media channels on Facebook, Twitter, and Instagram: observational study. Journal of Medical Internet Research, Toronto, v. 22, n. 9, p. e21204, 2020. DOI: https://doi.org/10.2196/21204. Disponível em: https://www.jmir.org/2020/9/e21204/. Acesso em: 20 set. 2021.

GALHARDI, Cláudia Pereira et al. Fato ou Fake? Uma análise da desinformação frente à pandemia da covid-19 no Brasil. Ciência \& Saúde Coletiva, Rio de Janeiro, v. 25, n. suppl 2, p. 4201-4210, 2020. DOI: https://doi.org/10.1590/1413-812320202510.2.28922020. Disponível em: https://www.scielo.br/j/csc/a/XnfpYRR 45Z4nXskC3PTnp8z/?lang=pt. Acesso em: 17 set. 2021.

GAO, Yankun et al. Electronic cigarette-related contents on Instagram: observational study and exploratory analysis. JMIR Public Health and Surveillance, Toronto, v. 6, n. 4, p. e21963, 2020. DOI: https://doi. org/10.2196/21963. Disponível em: https://publichealth.jmir.org/2020/4/e21963/. Acesso em: 20 set. 2021.

GIL-QUINTANA, Javier; SANTOVEÑA-CASAL, Sonia; ROMERO RIAÑO, Efrén. Realfooders influencers on Instagram: from followers to consumers. International Journal of Environmental Research and Public Health, Basel, v. 18, n. 4, p. 1624, 2021. DOI: https://doi.org/10.3390/ijerph18041624. Disponível em: https:// www.mdpi.com/1660-4601/18/4/1624. Acesso em: 20 set. 2021.

GREKIN, Emily R.; BEATTY, Jessica R.; ONDERSMA, Steven J. Mobile health interventions: exploring the use of common relationship factors. JMIR mHealth and uHealth, Toronto, v. 7, n. 4, p. e11245, 2019. DOI: https:// doi.org/10.2196/11245. Disponível em: https://mhealth.jmir.org/2019/4/e11245/. Acesso em: 17 set. 2021.

GUTIÉRREZ, Maria Gaby Rivero de; ALMEIDA, Ana Maria de. Outubro Rosa. Acta Paulista de Enfermagem, São Paulo, v. 30, n. 5, p. 3-5, 2017. DOI: https://doi.org/10.1590/1982-0194201700065. Disponível em: https:// www.scielo.br/j/ape/a/HcrYKxQsxZcQQbGSmw4RFCQ/?lang=pt. Acesso em: 17 set. 2021.

HABIBI, Sarah A.; SALIM, Lidya. Static vs. dynamic methods of delivery for science communication: a critical analysis of user engagement with science on social media. PloS One, São Francisco, v. 16, n. 3, p. e0248507, 2021. DOI: https://doi.org/10.1371/journal.pone.0248507. Disponível em: https://journals.plos.org/plosone/ article/comments?id=10.1371/journal.pone.0248507. Acesso em: 20 set. 2021.

HOUTS, Peter S. et al. The role of pictures in improving health communication: a review of research on attention, comprehension, recall, and adherence. Patient Education and Counseling, Limerick, v. 61, n. 2, p. 173-190, 2006. DOI: https://doi.org/10.1016/j.pec.2005.05.004. Disponível em: https://www.sciencedirect.com/ science/article/abs/pii/S0738399105001461?via\%3Dihub. Acesso em: 20 set. 2021.

HOUGHTON, Lauren C; HOWLAND, Renata E.; MCDONALD, Jasmine A. Mobilizing breast cancer prevention research through smartphone apps: a systematic review of the literature. Frontiers in Public Health, [s. I.], v. 7, p. 298, 2019. DOI: https://doi.org/10.3389/fpubh.2019.00298. Disponível em: https://www.frontiersin.org/ articles/10.3389/fpubh.2019.00298/full. Acesso em: 21 set. 2021.

INSTITUTO NACIONAL DE CÂNCER (INCA). Câncer de mama: é preciso falar disso. Rio de Janeiro: Inca, 2014.

INSTITUTO NACIONAL DE CÂNCER (INCA). Estimativa 2020: síntese de resultados e comentários. Rio de Janeiro: O Instituto, 2020. Disponível em: https://www.inca.gov.br/estimativa/sintese-de-resultados-ecomentarios. Acesso em: 10 mar. 2021.

INUMARU, Lívia Emi; SILVEIRA, Érika Aparecida da; NAVES, Maria Margareth Veloso. Fatores de risco e de proteção para câncer de mama: uma revisão sistemática. Cadernos de Saúde Pública, Rio de Janeiro, v. 27, n. 7, p. 1259-1270, 2011. DOI: https://doi.org/10.1590/S0102-311X2011000700002. Disponível em: https:// www.scielo.br/j/csp/a/ZbRRyNH4HRLXSbFNMms6RgM/abstract/?lang=pt\#: :text=As\%20pr\%C3\%A1ticas\%20 de $\% 20$ lacta $\%$ C3\%A7\%C3\%A30\%20e,bebida $\% 20$ alco\%C3\%B3lica\%2C\%20fator\%20de\%20risco. Acesso em: 20 set. 2021.

JENKINS, Eva L. et al. Strategies to improve health communication: can health professionals be heroes? Nutrients, Basel, v. 12, n. 6, p. 1861, 2020. DOI: https://doi.org/10.3390/nu12061861. Disponível em: https:// www.mdpi.com/2072-6643/12/6/1861. Acesso em: 20 set. 2021.

KATZ, Matthew S.; STALEY, Alicia C.; ATTAI, Deanna J. A History of \#BCSM and Insights for PatientCentered Online Interaction and Engagement. Journal of Patient-Centered Research and Reviews, Milwaukee, v. 7, n. 4, p. 304-312, 2020. DOI: https://doi.org/10.17294/2330-0698.1753. Disponível em: https:// institutionalrepository.aah.org/jpcrr/vol7/iss4/3/. Acesso em: 20 set. 2021. 
KAUR, Wandeep et al. Liking, sharing, commenting and reacting on Facebook: user behaviors' impact on sentiment intensity. Telematics and Informatics, [s. I.], v. 39, p. 25-36, 2019. DOI: https://doi.org/10.1016/j. tele.2018.12.005. Disponível em: https://www.sciencedirect.com/science/article/abs/pii/S0736585318304325. Acesso em: 20 set. 2021.

KEARNEY, Matthew D. et al. Characterizing HPV vaccine sentiments and content on Instagram. Health Education \& Behavior, Thousand Oaks, v. 46, n. 2 suppl, p. 37-48, 2019. DOI: https:// doi.org/10.1177\%2F1090198119859412. Disponível em: https://journals.sagepub.com/ doi/10.1177/1090198119859412. Acesso em: 20 set. 2021.

KICKBUSCH, Ilona; NUTBEAM, Don. A watershed for health promotion: the Shanghai Conference 2016. Health Promotion International, Eynsham, v. 32, n. 1, p. 2-6, 2017. DOI: https://doi.org/10.1093/heapro/ daw112. Disponível em: https://academic.oup.com/heapro/article/32/1/2/2979258. Acesso em: 17 set. 2021.

KIM, Cheonsoo; YANG, Sung-Un. Like, comment, and share on Facebook: how each behavior differs from the other. Public Relations Review, [s. I.], v. 43, n. 2, p. 441-449, 2017. DOI: https://doi. org/10.1016/j.pubrev.2017.02.006. Disponível em: https://www.sciencedirect.com/science/article/abs/pii/ S0363811116300157\#: :text=In\%20a\%20similar\%20way\%2C\%20Facebook,extra\%20commitment\%20or\%20 cognitive\%20effort. Acesso em: 20 set. 2021.

$\mathrm{KIM}$, Hankyul et al. Association between online information-seeking and adherence to guidelines for breast and prostate cancer screening. Preventing Chronic Disease, [s. I.], v. 15, n., 45, p. 1-11, 2018. DOI: http://dx.doi. org/10.5888/pcd15.170147. Disponível em: https://www.cdc.gov/pcd/issues/2018/17_0147.htm. Acesso em: 17 set. 2021.

KIM, Yunhwan; SONG, Donghwi; LEE, Yeon Ju. \#Antivaccination on Instagram: a computational analysis of hashtag activism through photos and public responses. International Journal of Environmental Research and Public Health, Basel, v. 17, n. 20, p. 7550, 2020. DOI: https://dx.doi.org/10.3390\%2Fijerph17207550. Disponível em: https://www.mdpi.com/1660-4601/17/20/7550. Acesso em: 20 set. 2021.

KITE, James et al. User perceptions of the make healthy normal campaign Facebook Page: a mixed methods study. Social Media + Society, [s. I.], v. 4, n. 3, p. 1-17, 2018. DOI: https:// doi.org/10.1177\%2F2056305118794639. Disponível em: https://journals.sagepub.com/ doi/10.1177/2056305118794639. Acesso em: 20 set. 2021.

LATHA, $\mathrm{K}$ et al. Effective use of social media platforms for promotion of mental health awareness. Journal of Education and Health Promotion, Mumbai, v. 9, p. 124, 2020. DOI: https://dx.doi.org/10.4103\%2Fjehp. iehp 90 20. Disponível em: https://www.jehp.net/article.asp?issn=2277-9531;year=2020;volume=9;issue=1;sp age $=124$ :epage=124; aulast=Latha. Acesso em: 21 set. 2021.

LATEEF, Nonam et al. Atrial fibrillation and cancer; understanding the mysterious relationship through a systematic review. Journal of Community Hospital Internal Medicine Perspectives, Filadéfia, v. 10, n. 2, p. 127-132, 2020. DOI: https://dx.doi.org/10.1080\%2F20009666.2020.1726571. Disponível em: https://www. tandfonline.com/doi/full/10.1080/20009666.2020.1726571. Acesso em: 17 set. 2021.

LEVAC, Joëlle J.; SULLIVAN, Tracey O. Social media and its use in health promotion. Revue interdisciplinaire des sciences de la santé, Ottawa, v. 1, n. 1, p. 47-53, 2010. DOI: https://doi.org/10.18192/ riss-ijhs.v1i1.1534. Disponível em: https://uottawa.scholarsportal.info/ojs/index.php/RISS-IJHS/article/ view/1534. Acesso em: 20 set. 2021.

LIMA, Marcela Sampaio et al. Temporal trend of cancer mortality in a Brazilian state with a medium Human Development Index (1980-2018). Scientific Reports, Londres, v. 10, 2020. DOI: https://doi.org/10.1038/ s41598-020-78381-4. Disponível em: https://www.nature.com/articles/s41598-020-78381-4. Acesso em: 17 set. 2021.

LUPTON, Deborah. Digital sociology. Nova York, NY: Routledge, 2015.

LUPTON, Deborah; MASLEN, Sarah. Telemedicine and the senses: a review. Sociology of Health \& IIIness, Oxford, v. 39, n. 8, p. 1557-1571, 2017. DOI: https://doi.org/10.1111/1467-9566.12617. Disponível em: https:// onlinelibrary.wiley.com/doi/10.1111/1467-9566.12617. Acesso em: 17 set. 2021.

MAJMUNDAR, Anuja et al. Public response to a social media tobacco prevention campaign: content analysis. JMIR Public Health and Surveillance, Toronto, v. 6, n. 4, p. e20649, 2020, doi: https://doi.org/10.2196/20649. Disponível em: https://publichealth.jmir.org/2020/4/e20649/PDF. Acesso em: 21 set. 2021. 
MARCON, Alessandro R.; BIEBER, Mark; AZAD, Meghan B. Protecting, promoting, and supporting breastfeeding on Instagram. Maternal \& Child Nutrition, Oxford, v. 15, n. 1, p. e12658, 2019. DOI: https://doi. org/10.1111/mcn.12658. Disponível em: https://onlinelibrary.wiley.com/doi/10.1111/mcn.12658. Acesso em: 20 set. 2021.

MASSEY, Phillip M. et al. Dimensions of misinformation about the HPV vaccine on Instagram: content and network analysis of social media characteristics. Journal of Medical Internet Research, Toronto, v. 22, n. 12, p. e21451, 2020. DOI: https://doi.org/10.2196/21451. Disponível em: https://www.jmir.org/2020/12/e21451. Acesso em: 20 set. 2021.

MERGEL, Ines. A framework for interpreting social media interactions in the public sector. Government Information Quarterly, [s. I.], v. 30, n. 4, p. 327-334, 2013. DOI: https://doi.org/10.1016/i.giq.2013.05.015. Disponível em: https://www.sciencedirect.com/science/article/abs/pii/S0740624X13000762. Acesso em: 20 set. 2021.

MIGOWSKI, Arn et al. Diretrizes para detecção precoce do câncer de mama no Brasil. II - Novas recomendações nacionais, principais evidências e controvérsias. Cadernos de Saúde Pública, Rio de Janeiro, v. 34, n. 6, p. e00074817, 2018. DOI: https://doi.org/10.1590/0102-311X00074817. Disponível em: https://www.scielo.brl//csp/a/8gGyb5s9Nt3nSsw5GFnnPQb/abstract/?lang=pt. Acesso em: 20 set. 2021.

MODAVE, François et al. Understanding perceptions and attitudes in breast cancer discussions on Twitter. Studies in Health Technology and Informatics, Amsterdã, v. 264, p. 1293-1297, 2019. DOI: https://doi. org/10.3233/shti190435. Disponível em: https://ebooks.iospress.nl/publication/52200. Acesso em: 20 set. 2021.

NGAI, Cindy Sing Bik et al. Grappling with the covid-19 health crisis: content analysis of communication strategies and their effects on public engagement on social media. Journal of Medical Internet Research, Toronto, v. 22, n. 8, p. e21360, 2020. DOI: https://dx.doi.org/10.2196\%2F21360. Disponível em: https://www. imir.org/2020/8/e21360/. Acesso em: 20 set. 2021.

NGAI, Cindy Sing Bik; SINGH, Rita Gill; LU, Wenze. Exploring drivers for public engagement in social media communication with medical social influencers in China. PloS One, São Francisco, v. 15, n. 10, p. e0240303, 2020. DOI: https://doi.org/10.1371/journal.pone.0240303. Disponível em: https://journals.plos.org/plosone/ article?id=10.1371/journal.pone.0240303. Acesso em: 20 set. 2021.

NOMAN, Muhammad Zaeem et al. Inhibition of Vps34 reprograms cold into hot inflamed tumors and improves anti-PD-1/PD-L1 immunotherapy. Science Advances, Washington, D. C., v. 6, n. 18, p. eaax7881, 2020. DOI: https://doi.org/10.1126/sciadv.aax7881. Disponível em: https://www.science.org/doi/10.1126/sciadv. aax7881. Acesso em: 17 set. 2021.

O'MAHONY, Máirín et al. Interventions for raising breast cancer awareness in women. Cochrane Database of Systematic Reviews, Oxford, v. 2, n. 2, p. CD011396, 2017. DOI: https://doi.org/10.1002/14651858.cd011396. pub2. Disponível em: https://www.cochranelibrary.com/cdsr/doi/10.1002/14651858.CD011396.pub2/full. Acesso em: 17 set. 2021.

OHL, Isabella Cristina Barduchi et al. Ações públicas para o controle do câncer de mama no Brasil: revisão integrativa. Revista Brasileira de Enfermagem, Brasília, DF, v. 69, n. 4, p. 793-803, 2016. DOI: https://doi.org/10.1590/0034-7167.2016690424i. Disponível em: https://www.scielo.br/j/reben/ a/6TL9tKq7vNXvkQRMsWrnyNv/abstract/?lang=pt. Acesso em: 17 set. 2021.

OMRANI, Atefeh et al. Breast education improves adolescent girls' breast knowledge, attitudes to breasts and engagement with positive breast habits. Frontiers in Public Health, Lausanne, v. 8, p. 591927, 2020. DOI: https://doi.org/10.3389/fpubh.2020.591927. Disponível em: https://www.frontiersin.org/articles/10.3389/ fpubh.2020.591927/full. Acesso em: 17 set. 2021.

ORGANIZAÇÃO MUNDIAL DA SAÚDE (OMS). The Ottawa Charter for Health Promotion. Ottawa: A Organização, 1986. Disponível em: https://www.who.int/publications/i/item/ottawa-charter-for-health-promotion. Acesso em: 17 set. 2021.

ORLANDINI, Leonardo Fleury et al. Epidemiological analyses reveal a high incidence of breast cancer in young women in Brazil. Journal of Global Oncology, Alexandria, VA, v. 7, p. 81-88, 2021. DOI: https://doi. org/10.1200/go.20.00440. Disponível em: https://ascopubs.org/doi/10.1200/GO.20.00440. Acesso em: 17 set. 2021. 
PAIGE, Samantha R. et al. Examining the relationship between online social capital and eHealth literacy: implications for Instagram use for chronic disease prevention among college students. American Journal of Health Education, Reston, v. 48, n. 4, p. 264-277, 2017. DOI: https://doi.org/10.1080/19325037.2017.131669 3. Disponível em: https://www.tandfonline.com/doi/abs/10.1080/19325037.2017.1316693?journalCode=ujhe20. Acesso em: 20 set. 2021.

PARK, Hyojung; REBER, Bryan H.; CHON, Myoung-Gi. Tweeting as health communication: health organizations' use of Twitter for health promotion and public engagement. Journal of Health Communication, Washington, D. C., v. 21, n. 2, p. 188-198, 2016. DOI: https://doi.org/10.1080/10810730.2015.1058435. Disponível em: https://www.tandfonline.com/doi/abs/10.1080/10810730.2015.1058435?journalCode=uhcm20. Acesso em: 20 set. 2021.

PASSOS, Kamilla Karla Maurício Passos et al. Quality of information about oral cancer in Brazilian Portuguese available on Google, Youtube, and Instagram. Medicina Oral Patología Oral y Cirugia Bucal, Valencia, v. 25, n. 3, p. e346-e352, 2020. DOI: https://doi.org/10.4317/medoral.23374. Disponível em: http://www.medicinaoral. com/medoralfree01/aop/23374.pdf. Acesso em: 17 set. 2021.

PEREIRA, Stephen P. et al. Early detection of pancreatic cancer. The Lancet. Gastroenterology \& Hepatology, Amsterdã, v. 5, n. 7, p. 698-710, 2020. DOI: https://doi.org/10.1016/s2468-1253(19)30416-9. Disponível em: https://www.thelancet.com/journals/langas/article/PIIS2468-1253(19)30416-9/fulltext. Acesso em: 17 set. 2021 .

PEYSER, Alexandra et al. Fertility education: what's trending on Instagram. Fertility Research and Practice, Londres, v. 7, n. 1, p. 3. 2021. DOI: https://doi.org/10.1186/s40738-021-00095-6. Disponível em: https:// fertilityresearchandpractice.biomedcentral.com/articles/10.1186/s40738-021-00095-6. Acesso em: 21 set. 2021.

PILGRIM, Katharina; BOHNET-JOSCHKO, Sabine. Selling health and happiness how influencers communicate on Instagram about dieting and exercise: mixed methods research. BMC Public Health, [s. I.], v. 19, p. 1054, 2019. DOI: https://doi.org/10.1186/s12889-019-7387-8. Disponível em: https://bmcpublichealth.biomedcentral. com/articles/10.1186/s12889-019-7387-8. Acesso em: 20 set. 2021.

PLACKETT, Ruth et al. Use of social media to promote cancer screening and early diagnosis: scoping review. Journal of Medical Internet Research, Toronto, v. 22, n. 11, p. e21582, 2020. DOI: https://doi. org/10.2196/21582. Disponível em: https://www.jmir.org/2020/11/e21582l. Acesso em: 20 set. 2021.

PRICHARD, Ivanka et al. The effect of Instagram \#fitspiration images on young women's mood, body image, and exercise behaviour. Body Image, [s. I.], v. 33, p. 1-6, 2020. DOI: https://doi. org/10.1016/j.bodyim.2020.02.002. Disponível em: https://www.sciencedirect.com/science/article/abs/pii/ S1740144519302578. Acesso em: 20 set. 2021.

R CORE TEAM. R: a language and environment for statistical computing. Vienna: R Foundation for Statistical Computing, 2015. Disponível em: https://www.r-project.orgl. Acesso em: 20 set. 2021.

RAMASWAMI, Ramya et al. Disparities in breast, lung, and cervical cancer trials worldwide. Journal of Global Oncology, Alexandria, VA, v. 4, p. 1-11, 2018. DOI: https://doi.org/10.1200/jgo.17.00226. Disponível em: https://ascopubs.org/doi/10.1200/JGO.17.00226. Acesso em: 17 set. 2021.

ROJAS, Kristin; STUCKEY, Ashley. Breast cancer epidemiology and risk factors. Clinical Obstetrics \& Gynecology, Hagerstown, v. 59, n. 4, p. 651-672, 2016. DOI: https://doi.org/10.1097/grf.0000000000000239. Disponível em: https://journals.Iww.com/clinicalobgyn/Abstract/2016/12000/Breast Cancer Epidemiology and Risk Factors.3.aspx. Acesso em: 17 set. 2021.

SANTOS, Dayane Evellyn et al. Efeito da radioterapia na função pulmonar e na fadiga de mulheres em tratamento para o câncer de mama. Fisioterapia e Pesquisa, São Paulo, v. 20, n. 1, p. 50-55, 2013. DOI: https://doi.org/10.1590/S1809-29502013000100009. Disponível em: https://www.scielo.br/j/fp/a/ bpXNGwB685fnGFX7QxJfKXz/abstract/?lang=pt. Acesso em: 17 set. 2021.

SANTOS, Manoel Antônio dos; SOUZA, Carolina de. Intervenções grupais para mulheres com câncer de mama: desafios e possibilidades. Psicologia: Teoria e Pesquisa, Brasília, DF, v. 35, p. e35410, 2019. DOI: https://doi.org/10.1590/0102.3772e35410. Disponível em: https://www.scielo.br/j/ptp/a/j8hnQ4ZkWMXGrnXsj7T Lt8p/?lang=pt. Acesso em: 17 set. 2021. 
SANZ-LORENTE, María et al. Web 2.0 tools in the prevention of curable sexually transmitted diseases: scoping review. Journal of Medical Internet Research, Toronto, v. 20, n. 3, p. e113, 2018. DOI: https://doi. org/10.2196/jmir.8871. Disponível em: https://www.jmir.org/2018/3/e113/. Acesso em: 20 set. 2021.

SARTORI, Amanda Caroline et al. Educational intervention using WhatsApp on medication adherence in hypertension and diabetes patients: a randomized clinical trial. Telemedicine and e-Health, Larchmont, v. 26, n. 12, p. 1526-1532, 2020. DOI: https://doi.org/10.1089/tmj.2019.0305. Disponível em: https://www.liebertpub. com/doi/10.1089/tmj.2019.0305. Acesso em: 17 set. 2021.

SELTZER, E. K. et al. Public sentiment and discourse about Zika virus on Instagram. Public Health, Londres, v. 150, p. 170-175, 2017. DOI: https://doi.org/10.1016/j.puhe.2017.07.015. Disponível em: https://www. sciencedirect.com/science/article/abs/pii/S0033350617302445?via\%3Dihub. Acesso em: 20 set. 2021.

SILVA, Karline Kelly da Silva et al. Estratégias de enfrentamento após o diagnóstico de câncer de mama. Revista Brasileira em Promoção da Saúde, Fortaleza, v. 33, p. 1-10, 2020. DOI: https://doi.org/10.5020/18 061230.2020.10022. Disponível em: https://periodicos.unifor.br/RBPS/article/view/10022. Acesso em: 17 set. 2021.

SILVA, Natally Regina Oliveira. Mortalidade por câncer de mama no Brasil de 2010 a 2019. Revista Científica Multidisciplinar Núcleo do Conhecimento, São Paulo, v. 13, n. 03, p. 116-125, 2021. Disponível em: https://www.nucleodoconhecimento.com.br/saude/no-brasil\#: :text=4.-,RESULTADOS, $44 \% 25 \% 20(9.917 \% 20$ \%C3\%B3bitos). Acesso em: 17 set. 2021.

KEMP, Simon. Digital 2021: the latest insights into the 'state of digital'. We Are Social, Nova York, 27 jan. 2021. Special Reports. Disponível em: https://wearesocial.com/blog/2021/01/digital-2021-the-latest-insightsinto-the-state-of-digital. Acesso em: 22 mar. 2021.

SMITH, Ben J.; TANG, Kwok Cho; NUTBEAM, Don. WHO Health Promotion Glossary: new terms. Health Promotion International, Eynsham, v. 21, n. 4, p. 340-345, 2006. DOI: https://doi.org/10.1093/heapro/dal033. Disponível em: https://academic.oup.com/heapro/article/21/4/340/688495. Acesso em: 17 set. 2021.

SOPIK, Victoria. International variation in breast cancer incidence and mortality in young women. Breast Cancer Research and Treatment, Dordrecht, v. 186, n. 2, p. 497-507, 2021. DOI: https://doi.org/10.1007/ s10549-020-06003-8. Disponível em: https://link.springer.com/article/10.1007\%2Fs10549-020-06003-8. Acesso em: 16 set. 2021.

SUNG, Hyuna et al. Global cancer statistics 2020: GLOBOCAN estimates of incidence and mortality worldwide for 36 cancers in 185 countries. CA: A Cancer Journal for Clinicians, Nova York, v. 71, n. 3, p. 209-249, 2021. DOI: https://doi.org/10.3322/caac.21660. Disponível em: https://acsjournals.onlinelibrary.wiley.com/ doi/10.3322/caac.21660. Acesso em: 16 set. 2021.

SYCINSKA-DZIARNOWSKA, Magdalena et al. Analysis of Instagram $®$ posts referring to cleft lip. International Journal of Environmental Research and Public Health, Basel, v. 17, n. 20, p. 7404, 2020. DOI: https:// dx.doi.org/10.3390\%2Fijerph17207404. Disponível em: https://www.mdpi.com/1660-4601/17/20/7404. Acesso em: 20 set. 2021

TELVIZIAN, Talar; MUKHERJI, Deborah. Germline mutations and prostate cancer: is it time to change treatment algorithms? Chinese Clinical Oncology, Hong Kong, v. 9, n. 5, p. 65, 2020. DOI: https://doi. org/10.21037/cco-19-207. Disponível em: https://cco.amegroups.com/article/view/50420/html. Acesso em: 17 set. 2021.

TENGLAND, Per-Anders. Behavior change or empowerment: on the ethics of health-promotion goals. Health Care Analysis, Dordrecht, v. 24, n. 1, p. 24-46, 2016. DOI: https://doi.org/10.1007/s10728-013-0265-0. Disponível em: https://link.springer.com/article/10.1007\%2Fs10728-013-0265-0. Acesso em: 17 set. 2021.

TORRE, Lindsey A. et al. Global cancer incidence and mortality rates and trends: an update. Cancer Epidemiology Biomarkers \& Prevention, Filadélfia, v. 25, n. 1, p. 16-27, 2016. DOI: https://doi. org/10.1158/1055-9965.epi-15-0578. Disponível em: https://cebp.aacrjournals.org/content/25/1/16. Acesso em: 17 set. 2021.

WANG, Yuxi et al. Systematic literature review on the spread of health-related misinformation on social media. Social Science \& Medicine, Oxford, v. 240, p. 112552, 2019. DOI: https://doi.org/10.1016/j. socscimed.2019.112552. Disponível em: https://www.ncbi.nlm.nih.gov/nlmcatalog/8303205. Acesso em: 20 set. 2021. 
WHITEHEAD, Dean; TAKET, Ann; SMITH, Pam. Action research in health promotion. Health Education Journal, [s. I.], v. 62, n. 1, p. 5-22, 2003. DOI: https://doi.org/10.1177\%2F001789690306200102. Disponível em: https://journals.sagepub.com/doi/abs/10.1177/001789690306200102?journalCode=heja. Acesso em: 17 set. 2021.

WILNER, Tamar; HOLTON, A. Breast cancer prevention and treatment: misinformation on Pinterest, 2018. American Journal of Public Health, New York, v. 110, n. suppl 3, p. S300-S304, 2020. DOI: https://dx.doi. org/10.2105\%2FAJPH.2020.305812. Disponível em: https://ajph.aphapublications.org/doi/full/10.2105/ AJPH.2020.305812. Acesso em: 20 set. 2021.

WOHN, Donghee Yvette; CARR, Caleb T.; HAYES, Rebecca A. How affective is a "like"?: the effect of paralinguistic digital affordances on perceived social support. Cyberpsychology, Behavior, and Social Networking, New Rochelle, v. 19, n. 9, p. 562-566, 2016. DOI: https://doi.org/10.1089/cyber.2016.0162. Disponível em: https://www.liebertpub.com/doi/10.1089/cyber.2016.0162. Acesso em: 20 set. 2021.

YAMAGUCHI, Miriam Ueda et al. O papel das mídias digitais e da literacia digital na educação não-formal em saúde (The role of digital media and digital literacy in non-formal health education). Revista Eletrônica de Educação, São Carlos, v. 14, p. 3761017, 2020. DOI: https://doi.org/10.14244/198271993761. Disponível em: http://www.reveduc.ufscar.br/index.php/reveduc/article/view/3761. Acesso em: 17 set. 2021.

YANG, Qinghua; VAN STEE, Stephanie K. The comparative effectiveness of mobile phone interventions in improving health outcomes: meta-analytic review. JMIR mHealth and uHealth, Toronto, v. 7, n. 4, p. e11244, 2019. DOI: https://doi.org/10.2196/11244. Disponível em: https://mhealth.jmir.org/2019/4/e11244/. Acesso em 17 set. 2021.

YANG, Yi et al. Promoting public engagement during the covid-19 crisis: how effective is the Wuhan local government's information release?. International Journal of Environmental Research and Public Health, [s. I.], v. 18, n. 1, p. 118, 2020. DOI: https://doi.org/10.3390/ijerph18010118. Disponível em: https://www.mdpi. com/1660-4601/18/1/118. Acesso em: 20 set. 2021.

YOULDEN, Danny R. et al. The descriptive epidemiology of female breast cancer: an international comparison of screening, incidence, survival and mortality. Cancer Epidemiology, Amsterdã, v. 36, n. 3, p. $237-248,2012$. DOI: https://doi.org/10.1016/j.canep.2012.02.007. Disponível em: https://www.sciencedirect.com/science/ article/abs/pii/S187778211200029X?via\%3Dihub. Acesso em: 17 set. 2021.

YUKI, Tania. What makes brands' social content shareable on Facebook? Journal of Advertising Research, Oxford, v. 55, n. 4, p. 458-470, 2015. DOI: http://dx.doi.org/10.2501/JAR-2015-026. Disponível em: http://www. journalofadvertisingresearch.com/content/55/4/458. Acesso em: 20 set. 2021.

ZARZYCKI, Nick. Reach vs. impressions: what's more important to track? Hootsuite, [s. I.], 26 jun. 2018. Strategy. Disponível em: https://blog.hootsuite.com/reach-vs-impressions/. Acesso em: 5 abr. 2021.

ZAVATTARO, Staci M.; SEMENTELLI, Arthur J. A critical examination of social media adoption in government: introducing omnipresence. Government Information Quarterly, [s. I.], v. 31, n. 2, p. 257-264, 2014. DOI: https://doi.org/10.1016/j.giq.2013.10.007. Disponível em: https://www.sciencedirect.com/science/article/abs/pii/ S0740624X14000045. Acesso em: 20 set. 2021. 